

\section{Gestão Editorial de Periódicos Científicos: tendências e boas práticas}




\section{Gestão Editorial de Periódicos Científicos: tendências e boas práticas}

Organizadores

Lúcia da Silveira

Fabiano Couto Côrrea da Silva

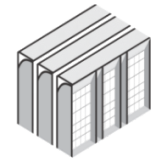

PU B I ICACÓES

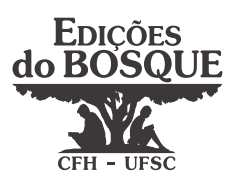

$1^{a}$ edição | 2020 


\section{(ㄷ)(1)}

Esta obra está sob a licença Creative Commons Atribuição 4.0. Para mais informações acesse: $<$ https://creativecommons.org/licenses/by/4.0/>.

\author{
Organização \\ Lúcia da Silveira \\ Fabiano Couto Côrrea da Silva \\ Conselho Editorial - BU Publicações \\ Roberta Moraes de Bem \\ Andréa Figueiredo Leão Grants \\ José Paulo Speck Pereira \\ Luciana Bergamo Marques \\ Cristiano Motta Antunes \\ Comissão científica \\ Anna Khris Furtado Dutra \\ Anderson Mendes \\ Andréa Figueiredo Leão Grants \\ Clarissa Agostini Pereira \\ Gabriel Araldi Walter \\ Fabiano Couto Côrrea da Silva \\ Jorge Moisés Kroll do Prado \\ Juliana Aparecida Gulka \\ Lúcia da Silveira \\ Maria Bernardete Martins Alvez \\ Revisão ortográfica e gramatical \\ Zulma Neves de Amorim Borges
}

\section{Normalização}

Zulma Neves de Amorim Borges

Revisão Geral

Andréa Figueiredo Leão Grants Juliana Aparecida Gulka

Lúcia da Silveira

Arte visual

Lara Benedet

Pablo Fiqueiredo

Cristiano Motta Antunes

Diagramação

Arnoldo Bublitz

BU Publicações UFSC

Campus Universtário Reitor João

David F. Lima, Acesso Trindade.

Florianópolis, SC

conselhoeditorial.bu@contato.ufsc.br

+554837219310

Edições do Bosque

Nuppe/CFH/UFSC

https://nuppe.ufsc.br

https://doi.org/10.5007/978-65-87206-08-0

Catalogação na fonte pela Biblioteca Universitária da Universidade Federal de Santa Catarina

G393

Gestão editorial de periódicos científicos [recurso eletrônico] : tendências e boas práticas / organizadores, Lúcia da Silveira, Fabiano Couto Côrrea da Silva. - 1. ed. - Florianópolis : BU Publicações/UFSC : Edições do Bosque/UFSC, 2020.

226 p. : il., gráf., tab.

ISBN 978-65-87206-08-0

E-book (PDF).

1. Periódicos eletrônicos. 2. Editores de periódicos. 3. Tecnologia - Serviços de informação. 4. Ciência da Informação. I. Silveira, Lúcia. II. Silva, Fabiano Couto Côrrea da.

CDU 001:655.52

Elaborada pelos bibliotecários Jonathas Troglio e Suélen Andrade - CRB-14/1093 e CRB-14/1666 


\section{Sumário}

Apresentação

Prefácio - Un libro para explicar los marcos y los retos de las revistas científicas Ernest Abadal

01 - Gestão editorial: tendências e desafios na transição para a ciência aberta

Solange M. dos Santos e Lilian N. Calò

02 - Gestão de dados científicos para periódicos

Fabiano Couto Corrêa da Silva

03 - Direitos de autor e licenças Creative Commons para periódicos científicos de acesso aberto

Fabio Lorensi Canto, Enrique Muriel-Torrado e Adilson Luiz Pinto

04 - Como pensar a acessibilidade em artigos de periódicos: tendências em design universal para pessoas com deficiência visual.................105 José Carlos Rodrigues e Salete Cecília de Souza

05 - Ferramentas de gestão editorial para periódicos em acesso aberto........................................................................... 127 Juliana Fachin, Lúcia da Silveira e Diego Abadan

06 - Dez boas práticas para portais de periódicos Lúcia da Silveira, Gildenir Carolino Santos e Claudia Oliveira de Moura Bueno

07 - Marketing científico digital e práticas de comunicação e divulgação de portais de periódicos: notas de uma primeira incursão. 197 Ronaldo Ferreira de Araújo, Celsiane Aline Vieira Araújo, Sergio Dias Cirino, Marcella Ximenes de Mello Boehler e Renata Kelly de Arruda 


\section{Prefácio}

\section{Un libro para explicar los marcos y los retos de las revistas científicas}

Ernest Abadal

Las revistas científicas aparecen a finales del s. XVII en París y Londres, en el seno de las primeras sociedades científicas como instrumento para facilitar la comunicación entre científicos y teniendo el peer review como sistema de control de la calidad de sus contenidos. Durante más de 300 años, pues, las revistas científicas han servido para difundir los progresos científicos y para reconocer las autorías de estos avances sin apenas alteraciones ni sobresaltos en su estructura ni funcionamiento.

Ahora bien, a partir de 1990 se han ido introduciendo de manera escalonada tres grandes cambios que se han erigido en marcos estructurales que han condicionado totalmente el funcionamiento de las revistas científicas. Utilizamos aquí el término "marco" en el sentido definido por Lakoff en su famoso libro Don't think of an elephant (2004), en el cual se pone de manifiesto la importancia de establecer un marco o encuadre sobre la realidad porque el marco es el contexto de interpretación que condiciona la actuación de todas las personas respecto de esta realidad.

Frames are mental structures that shape the way we see the world. As a result they shape the goals we seek, the plans we make, the way we act, and what counts as a good or bad outcome of our actions. In politics our frames shape our social policies and the institutions we form to carry out policies. (LAKOFF, 2004).

Así pues, las actuaciones de los editores cuando planifican los nuevos ejemplares de las revistas o diseñan determinadas propuestas editoriales, así como también las acciones de los autores cuando preparan sus originales para ser enviados a las revistas, están siempre condicionadas por el marco o encuadre predominante. Es importante, por tanto, que tanto editores como autores conozcan con detalle estos marcos y los retos que se derivan para poder aprovechar al máximo la función de las revistas como instrumento de difusión de la investigación. 


\section{Los marcos}

La primera gran transformación para las revistas tiene lugar a mediados de 1990 y fue la digitalización de sus contenidos. La posibilidad de difundir los artículos en formato digital alteró sustancialmente no tan sólo el sistema de producción --rebajando los costes- sino también la distribución, ya que facilitó que los contenidos llegaran de manera inmediata a cualquier parte del mundo creando una audiencia global. La segunda transformación se inicia a partir de 2000 cuando aparece el acceso abierto, el nuevo modelo de comunicación científica basado en la difusión libre y gratuita de los contenidos académicos. Este cambio afectó no solo al sistema de difusión (los lectores ya no tendrán barreras para la consulta de los contenidos), sino que también ha modificado el modelo económico que sostiene el mercado de las revistas científicas (han aparecido nuevos editores, se han creado repositorios, se ha trasladado el coste del lector al autor, etc.). Finalmente, a partir de 2015, con la consolidación del concepto de ciencia abierta, se empieza a perfilar un nuevo modelo de investigación que supone aplicar la "apertura" no tan solo a la fase de difusión de la ciencia sino también a la recogida de datos (que deben ser "abiertos"), a la revisión por expertos, a la colaboración ciudadana, etc.

Como hemos visto, estos cambios se han producido de manera ordenada y cada uno ha facilitado el surgimiento del siguiente. Se trata de un crescendo coordinado ya que cada uno de ellos ha ido completando y profundizando los pasos del anterior y no podría haberse realizado sin ellos. Así pues, para poder establecer el acceso abierto era imprescindible disponer de las revistas en formato digital y, por otro lado, la ciencia abierta se ha dibujado a partir de las bases del acceso abierto, que se han ampliado a todas las etapas de la investigación científica.

En resumen, en menos de 30 años han sido tres los "marcos" que han encuadrado el funcionamiento de las revistas científicas y que han obligado a que todas ellas se adaptaran a sus características y condicionamientos: la digitalización, el acceso abierto y la ciencia abierta. En cualquier caso, no se puede olvidar que actualmente todos los editores y también los autores están totalmente condicionados por estos marcos y han debido (o deben) adaptarse a ellos para sobrevivir en el nuevo ecosistema de la comunicación científica. 


\section{Los retos}

Dibujados los marcos contextuales, podemos bajar un peldaño y establecer cuáles son, en nuestra opinión, los retos actuales de las revistas científicas. Se podría hacer referencia a un buen número de ellos, tal y como detallamos en la monografía Revistas científicas: situación actual y retos de futuro (ABADAL, 2017) pero vamos a concentrarnos tan sólo en cinco.

La sostenibilidad económica se encuentra en la base estructural de las revistas científicas de acceso abierto. Una vez que los ingresos ya no pueden reposar en las suscripciones de los lectores, se deben encontrar vías de financiación para las tareas editoriales que, como es sabido, provendrán fundamentalmente del pago de una tasa por parte de los autores o de la financiación pública a las revistas (por parte de las universidades, centros de investigación, etc.).

Asegurar la calidad de los contenidos también es otro de los retos, especialmente en el contexto actual de los "predatory journals", de las revistas que dejan de lado la revisión por expertos y que publican todo lo que reciben a cambio de tasas elevadas. Estas publicaciones no tienen nada que ver con el acceso abierto pero han extendido sospechas sobre las revistas open access que deben ser combatidas con los hechos. Las revistas deben proceder con la máxima transparencia y el rigor editorial más absoluto para disipar de manera inmediata y sin contestación la sombra de malas prácticas.

La revisión abierta también se irá abriendo camino y habrá que ver cómo se encaja con el funcionamiento de los procesos editoriales. El open peer review supone incorporar una visión abierta al proceso de revisión por expertos de los contenidos científicos (especialmente los artículos de revista). En este sentido, todas las acciones que vayan en el camino de aumentar la transparencia lo favorecerán, ya sea mostrando las identidades de los autores y los revisores, poniendo los informes de los revisores al alcance de los lectores y, en último término, facilitando una participación más amplia en el proceso de revisión.

Los datos abiertos constituyen uno de los elementos clave de la ciencia abierta. Se trata de conseguir que los datos científicos sean no tan sólo recogidos, sino etiquetados con metadatos y almacenados para que pueda ser posible su recuperación y eventual reutilización por parte de otros científicos interesados. La recomendación actual solicita que los datos estén abiertos por defecto y tan solo 
estén cerrados cuando esté debidamente justificado. Las revistas científicas deberán encontrar acomodo a estos datos de investigación, para que puedan acompañar a los textos de los artículos. En estos momentos ya se pueden encontrar diversas fórmulas, aunque la más utilizada quizá sea la de pedir el depósito de datos en plataformas como Zenodo y establecer el enlace del artículo publicado con los datos.

Finalmente, nos vamos a referir a uno de los retos de mayor calado: el cambio de modelo de evaluación de los artículos y de las revistas. La necesidad de modificar los criterios para la evaluación de la investigación y de las publicaciones ha constituido una demanda desde hace años que estalló con diversos pronunciamientos públicos en los que destaca la San Francisco Declaration on Research Assessment (2012) y también el Leiden Manifesto (HICKS et al., 2015). Se trata de declaraciones muy críticas con el monopolio ejercido hasta ahora por el factor de impacto para evaluar las publicaciones y que sugieren, entre otras medidas, incorporar puntos de vista cualitativos, valorar a nivel de artículo (y no con el factor de impacto de la revista) y también ampliar el espectro de medidas a incorporar a cada publicación. Se destaca que no se debe poner tanto énfasis en el factor de impacto y se pone atención a la emergencia de métricas alternativas y se aboga por el uso de unas métricas responsables que sean robustas, humildes, transparentes, diversas y reflexivas.

\section{El libro}

Hemos descrito los marcos y también los retos. Para ser un buen editor de revistas, no obstante, no es suficiente conocer el contexto teórico. Existen también un sinfín de cuestiones relacionadas con el día a día de los procesos con las que el editor y el autor deben lidiar continuamente: la selección de originales, la revisión, la corrección, la maquetación, la publicación en portales web y la distribución en redes sociales, entre otros.

Gestao editorial de periódicos científicos, el libro que prologamos, incide en todas estas cuestiones antes descritas, no tan sólo en las teóricas sino también en las prácticas. El libro profundiza y describe a la perfección el contexto teórico que hemos dibujado. Dispone de capítulos en los cuales se tratan con detalle los dos marcos actuales de las revistas científicas, tanto el acceso abierto como la ciencia 
abierta. También incluye otros capítulos en los cuales se hace referencia a los retos antes apuntados, ya sea la incorporación de datos abiertos en los artículos, o la revisión abierta, entre otros. Además de ello, se presenta información detallada sobre aspectos prácticos relacionados con la gestión editorial de las revistas, ya sean los instrumentos (ferramentas) y plataformas tecnológicas a utilizar, el uso de licencias CreativeCommons, las estrategias de divulgación, la adaptación de los artículos a personas con deficiencias visuales, etc.

No son abundantes los estudios monográficos sobre revistas científicas. El público potencial para este libro es amplio y diverso, ya que no solo incluye a los editores de revistas académicas, sino que también puede ser de interés para los científicos y académicos de cualquier disciplina que tienen actividad como autores. Por otro lado, no se puede olvidar a los bibliotecarios, en especial los más implicados en las tareas de apoyo a la investigación ya que deberán orientar a estos mismos autores y editores en el siempre variante entorno de la comunicación científica. El carácter práctico y aplicado de este libro puede ser de mucha utilidad a todos ellos.

Quiero también destacar el hecho de que estamos delante de una obra colectiva en el sentido más literal de la palabra. Son casi veinte los autores participantes, aunque más bien debería decir "las autoras" porque las mujeres son mayoría y creo que es un detalle que se debe destacar. Estas personas proceden de distintas universidades brasileñas y tienen diversas especializaciones que han permitido disponer de voces expertas para cada uno de los capítulos.

En resumen, para interaccionar con las revistas científicas, ya sea como autor o editor, es fundamental conocer el marco y los retos que las condicionan, así como los aspectos prácticos del funcionamiento diario. Este libro da respuesta a todo ello. Se trata de una obra muy recomendable que puede ser una referencia en esta temática durante años. 


\section{Referencias}

ABADAL, E. (ed.). Revistas científicas: situación actual y retos de futuro. Barcelona: Publicacions i Edicions de la UB, 2017.

HICKS, D. et al. The Leiden Manifesto for research metrics. Nature, [s. l.], v. 520, p. 429-431, 2015. Disponível em: https://www.nature.com/news/ bibliometrics-the-leiden-manifesto-for-research-metrics-1.17351. Acesso em: 5 maio 2019.

LAKOFF, G. Don't think of an elephant! know your values and frame the debate: the essential guide for progressives. Vermont, USA: White River Junction, Chelsea Green Publishing, 2004.

SAN FRANCISCO DECLARATION OF RESEARCH ASSESSMENT (DORA). San Francisco, 2012. Disponível em: https://sfdora.org/read/es/. Acesso em: 5 maio 2019. 